\title{
Efficient Cropping Zone for Groundnut in Tamil Nadu
}

\author{
S. Sanbagavalli, R. Tamilmozhi* and R. Karthikeyan \\ Department of Agronomy, Tamil Nadu Agricultural University, Coimbatore, India \\ *Corresponding author
}

A B S T R A C T

\section{Keywords}

Ground nut, Relative spread index, Relative yield index, Efficient cropping zone

Article Info

Accepted:

15 October 2020

Available Online:

10 November 2020
An analytical study was made to identify the efficient cropping zone for groundnut in Tamil Nadu. For this study the data on percentage area and productivity of groundnut for ten years (2006-07 to 2015-16) were collected. Based on relative spread index (RSI) and relative yield index (RYI) the efficient cropping zone were identified. Among thirty one districts, five districts were found as most efficient cropping zones (MECZ).

\section{Introduction}

In Tamil Nadu, Groundnut was cultivated in 3.47 Lakh ha area with the average productivity of $2574 \mathrm{~kg} \mathrm{ha}^{-1}$ (Season and Crop Report, 2015-16). Even though there was a wider spread of cultivated area in different regions have less contribution on total production, because of lower productivity. The productivity of crops was determined by their adaptability to the particular region. To achieve sustainability, the cultivation of crops should be carried on their potential zones. This study assists to found the efficient cropping zone for Groundnut in Tamil Nadu Which helps to get higher yield in limited area through better crop selection and also the development of new technologies which enhances the productivity still further.

\section{Materials and Methods}

The data on percent area and productivity of groundnut for different districts of Tami Nadu were collected from the season and crop report by the Department of Economics and Statistics, Government of Tamil Nadu. From this ten year (2006-07 to 2015-16) data, relative spread index (RSI) and relative yield index (RYI) were computed by using Kanwar (1972) formula. 
RSI $=\frac{\text { Area of that particular crop expressed as \% of total cultivable area in the district }}{\text { Area of that particular crop expressed as \% of total cultivable area in the state }} \times 100$

RYI $=\frac{\text { Mean yield of a particular crop in a district }}{\text { Mean yield of a particular crop in the state }} \times 100$

For each year, the RSI and RYI were calculated separately. Based on the criteria for efficient cropping zone (Table 1), the efficient cropping zone for groundnut crop among all districts were identified.

\section{Results and Discussion}

Relative spread index (RSI): The data of relative spread index for groundnut (Table 2) indicated that among all districts, the RSI was maximum (397) with Thiruvannamalai district followed by Vellore district (304). Higher RSI indicates the major groundnut cultivating area. The soil type, rainfall pattern and other climatic factors of that area was highly favoured for groundnut cultivation.
Among all other districts The Nilgiris (0.14) have lowest RSI value followed by Kanyakumari (0.33), which indicated the lower spread of groundnut due to unsuitable soil and climatic conditions.

Relative yield index (RYI): The relative yield index (Table 3) was higher in Thiruvallur (179) followed by Thiruvarur (171) district. Higher RYI shows those areas have higher productivity of groundnut.

Efficient cropping zone: From the computed data (Table 4), it could be interpreted that out of thirty one districts, Kancheepuram and Vilupuram districts have high RSI \& high RYI.

Thiruvallur and Karur districts have medium RSI with high RYI. These four districts were categorized under Most Efficient Cropping Zone (MECZ) for groundnut. These districts played a major role for groundnut production in Tamil Nadu.

Table.1 Criteria for efficient cropping zone

\begin{tabular}{|c|c|c|}
\hline RSI & RYI & Cropping Zone \\
\hline High/Medium & High & Most Efficient Cropping Zone (MECZ) \\
\hline $\begin{array}{l}\text { High/Medium/Low } \\
\text { Low } \\
\text { High/Medium }\end{array}$ & $\begin{array}{l}\text { Medium } \\
\text { High } \\
\text { Low }\end{array}$ & $\begin{array}{l}\text { Efficient Cropping Zone (ECZ) } \\
\text { Not Efficient Cropping Zone (NECZ) }\end{array}$ \\
\hline Low & Low & Highly Inefficient Cropping Zone (HICZ) \\
\hline $\begin{array}{l}\text { Relative Spread In } \\
>125 \text { High } \\
\text { 75-125 Medium } \\
\text { <75 Low }\end{array}$ & $\begin{array}{l}\text { x }(\mathrm{RSI}) \mathrm{Re} \\
>100 \mathrm{Hi} \\
75-100 \mathrm{~N} \\
<75 \mathrm{Low}\end{array}$ & Yield Index (RYI) \\
\hline
\end{tabular}


Table.2 Percentage area of groundnut

\begin{tabular}{|c|c|c|c|c|c|c|c|c|c|c|c|c|}
\hline District & 2006-07 & 2007-08 & 2008-09 & 2009-10 & 2010-11 & 2011-12 & 2012-13 & 2013-14 & 2014-15 & $2015-16$ & Ave. & RYI \\
\hline Kancheepuram & 4149 & 2997 & 3584 & 4187 & 4469 & 3823 & 3580 & 3756 & 3600 & 3089 & 3723 & 158 \\
\hline Thiruvallur & 3734 & 3480 & 3850 & 4281 & 3518 & 4094 & 4584 & 5089 & 4823 & 4562 & 4202 & 179 \\
\hline Cuddalore & 3193 & 1855 & 2535 & 2918 & 3093 & 3569 & 3637 & 3880 & 4663 & 6469 & 3581 & 152 \\
\hline Vilupuram & 2076 & 2402 & 2447 & 2897 & 2392 & 2728 & 2719 & 3265 & 3100 & 2966 & 2699 & 115 \\
\hline Vellore & 1665 & 1885 & 1730 & 1924 & 2265 & 2257 & 2123 & 2564 & 2768 & 3064 & 2225 & 95 \\
\hline Thiruvannamalai & 1825 & 2139 & 1503 & 1823 & 1849 & 2739 & 2119 & 2463 & 2419 & 1732 & 2061 & 88 \\
\hline Salem & 1511 & 1454 & 1497 & 1539 & 2192 & 2484 & 1932 & 1989 & 2395 & 2158 & 1915 & 81 \\
\hline Namakkal & 1732 & 1660 & 1902 & 1569 & 2340 & 2438 & 1365 & 2110 & 2171 & 1764 & 1905 & 81 \\
\hline Dharmapuri & 1945 & 2282 & 2385 & 1819 & 2028 & 3193 & 2226 & 2460 & 2798 & 2763 & 2390 & 102 \\
\hline Krishnagiri & 1189 & 2109 & 1563 & 1790 & 2374 & 3088 & 2112 & 2666 & 2104 & 2271 & 2127 & 90 \\
\hline Coimbatore & 1689 & 1878 & 3304 & 1757 & 2033 & 2528 & 1573 & 2451 & 2162 & 2256 & 2163 & 92 \\
\hline Thiruppur & - & - & 1888 & 2272 & 1952 & 2388 & 1253 & 1416 & 2259 & 2026 & 1932 & 82 \\
\hline Erode & 1371 & 1609 & 2141 & 1695 & 1861 & 1406 & 1405 & 1862 & 1885 & 1738 & 1697 & 72 \\
\hline Thiruchirappalli & 2258 & 1846 & 1609 & 1748 & 1806 & 2337 & 2527 & 2538 & 2467 & 2180 & 2132 & 91 \\
\hline Karur & 2258 & 2280 & 2432 & 2430 & 1858 & 2634 & 2713 & 3208 & 2467 & 2792 & 2507 & 107 \\
\hline Perambalur & 1924 & 1287 & 1492 & 2144 & 1385 & 2089 & 2489 & 2636 & 2518 & 2838 & 2080 & 88 \\
\hline Ariyalur & - & - & - & 1946 & 3091 & 4134 & 2527 & 4290 & 4273 & 4217 & 3497 & 149 \\
\hline Pudukkottai & 1759 & 1188 & 1283 & 1685 & 1564 & 2175 & 1967 & 2032 & 3344 & 3184 & 2018 & 86 \\
\hline Thanjavur & 2327 & 1414 & 2220 & 3627 & 3760 & 3153 & 3103 & 3714 & 3788 & 4582 & 3169 & 135 \\
\hline Thiruvarur & 2549 & 2199 & 2009 & 3634 & 5041 & 5629 & 3218 & 5225 & 4949 & 5687 & 4014 & 171 \\
\hline Nagapattinam & 3800 & 1985 & 2517 & 3017 & 3164 & 3840 & 2872 & 3211 & 3252 & 3719 & 3138 & 133 \\
\hline Madurai & 2132 & 1871 & 1981 & 1536 & 1828 & 1900 & 2000 & 2146 & 2124 & 1716 & 1923 & 82 \\
\hline Theni & 2149 & 1971 & 1793 & 2197 & 1906 & 2452 & 5213 & 2442 & 3501 & 3384 & 2701 & 115 \\
\hline Dindigul & 2387 & 2203 & 3213 & 2594 & 2859 & 3510 & 2503 & 2940 & 3867 & 2797 & 2887 & 123 \\
\hline RamanathaPuram & 1246 & 771 & 1486 & 1336 & 1328 & 1390 & 1252 & 946 & 2254 & 1359 & 1337 & 57 \\
\hline Virudhunagar & 1573 & 1183 & 1291 & 678 & 1096 & 1671 & 1109 & 1531 & 1598 & 2150 & 1388 & 59 \\
\hline Sivagangai & 1226 & 838 & 1470 & 909.6 & 1186 & 2680 & 2542 & 1817 & 1389 & 1520 & 1558 & 66 \\
\hline Thirunelveli & 1668 & 1767 & 1755 & 1501 & 2212 & 3027 & 2614 & 2762 & 3119 & 2841 & 2327 & 99 \\
\hline Thoothukudi & 1461 & 1598 & 1302 & 1389 & 2227 & 2940 & 2602 & 2999 & 3049 & 2926 & 2249 & 96 \\
\hline The Nilgiris & - & - & - & 1400 & 1882 & - & - & 2213 & 1000 & 2175 & 1734 & 74 \\
\hline Kanyakumari & 1583 & 1641 & 1500 & 1450 & 1882 & 2202 & 1891 & 2870 & 2786 & 2574 & 2038 & 87 \\
\hline District Average & 1981 & 1957 & 1990 & 2169 & 2323 & 2751 & 2314 & 2721 & 2753 & 2574 & 2353 & \\
\hline
\end{tabular}


Table.3 Productivity of groundnut

\begin{tabular}{|c|c|c|c|c|c|c|c|c|c|c|c|c|}
\hline District & 2006-07 & 2007-08 & 2008-09 & 2009-10 & 2010-11 & 2011-12 & 2012-13 & 2013-14 & 2014-15 & 2015-16 & Ave. & RSI \\
\hline Kancheepuram & 14.53 & 15.95 & 16.18 & 13.78 & 12.32 & 12.87 & 14.17 & 10.35 & 11.3 & 8.41 & 12.99 & 184 \\
\hline Thiruvallur & 10.45 & 12.21 & 10.84 & 9.15 & 7.60 & 7.43 & 7.80 & 6.07 & 4.6 & 4.71 & 8.086 & 115 \\
\hline Cuddalore & 5.18 & 5.62 & 5.42 & 4.65 & 3.33 & 3.24 & 4.01 & 3.59 & 3.4 & 2.90 & 4.134 & 59 \\
\hline Vilupuram & 13.09 & 14.78 & 13.83 & 13.22 & 10.81 & 10.20 & 10.14 & 9.09 & 7.0 & 7.03 & 10.92 & 155 \\
\hline Vellore & 24.37 & 24.73 & 24.09 & 22.67 & 22.25 & 21.66 & 20.27 & 18.65 & 18.6 & 17.10 & 21.44 & 304 \\
\hline Thiruvannamalai & 31.97 & 34.76 & 36.45 & 29.25 & 24.19 & 22.79 & 24.10 & 25.47 & 27.6 & 23.29 & 27.99 & 397 \\
\hline Salem & 12.52 & 11.24 & 9.85 & 8.80 & 7.18 & 6.05 & 6.28 & 6.43 & 6.8 & 7.16 & 8.23 & 117 \\
\hline Dharmapuri & 7.57 & 7.71 & 6.48 & 6.30 & 5.08 & 6.84 & 6.70 & 5.28 & 4.0 & 4.61 & 6.06 & 86 \\
\hline Krishnagiri & 9.88 & 8.97 & 9.33 & 8.67 & 7.93 & 7.50 & 7.53 & 5.10 & 5.5 & 5.89 & 7.63 & 108 \\
\hline Coimbatore & 4.73 & 4.65 & 4.32 & 3.83 & 3.59 & 3.82 & 3.14 & 2.86 & 2.6 & 2.75 & 3.63 & 51 \\
\hline Thiruppur & - & - & 4.79 & 4.04 & 4.00 & 4.13 & 3.23 & 4.09 & 4.1 & 4.58 & 4.12 & 58 \\
\hline Erode & 10.65 & 10.19 & 10.46 & 8.63 & 8.98 & 9.32 & 9.09 & 9.34 & 10.1 & 10.55 & 9.73 & 138 \\
\hline Thiruchirappalli & 7.08 & 7.35 & 6.80 & 6.37 & 5.85 & 6.44 & 5.69 & 4.77 & 4.8 & 5.28 & 6.04 & 86 \\
\hline Karur & 3.99 & 6.22 & 7.57 & 7.40 & 6.98 & 6.95 & 5.35 & 2.86 & 4.9 & 4.98 & 5.72 & 81 \\
\hline Ariyalur & - & - & 12.54 & 12.52 & 10.47 & 12.24 & 9.43 & 8.80 & 8.0 & 7.90 & 10.24 & 14 \\
\hline Pudukkottai & 15.16 & 15.46 & 12.54 & 12.62 & 10.95 & 11.08 & 8.73 & 6.40 & 4.9 & 6.46 & 10.43 & 148 \\
\hline Thanjavur & 2.47 & 5.69 & 3.34 & 3.20 & 2.81 & 2.90 & 2.97 & 1.88 & 1.5 & 1.80 & 2.86 & 40 \\
\hline Thiruvarur & 0.84 & 3.00 & 0.47 & 0.73 & 0.71 & 1.46 & 1.70 & 1.13 & 0.5 & 0.65 & 1.12 & 16 \\
\hline Nagapattinam & 1.16 & 1.46 & 1.24 & 0.89 & 0.94 & 0.93 & 0.78 & 0.68 & 0.6 & 0.64 & 0.93 & 13 \\
\hline Madurai & 3.48 & 3.10 & 3.04 & 2.50 & 2.15 & 2.35 & 2.68 & 1.90 & 2.0 & 1.85 & 2.51 & 35 \\
\hline Theni & 1.70 & 1.58 & 1.95 & 1.51 & 2.12 & 1.75 & 1.51 & 1.50 & 1.5 & 1.93 & 1.71 & 24 \\
\hline Dindigul & 5.09 & 6.01 & 4.70 & 3.75 & 4.72 & 5.16 & 3.39 & 2.10 & 3.4 & 4.44 & 4.28 & 61 \\
\hline RamanathaPuram & 3.72 & 3.26 & 2.91 & 5.59 & 2.06 & 1.98 & 2.00 & 1.78 & 1.6 & 1.92 & 2.68 & 38 \\
\hline Virudhunagar & 5.07 & 5.71 & 6.28 & 3.38 & 4.62 & 4.20 & 3.84 & 4.29 & 4.6 & 4.44 & 4.64 & 66 \\
\hline Sivagangai & 4.35 & 4.64 & 3.66 & 0.78 & 3.43 & 5.16 & 3.41 & 3.32 & 2.3 & 3.12 & 3.42 & 48 \\
\hline Thirunelveli & 2.05 & 1.59 & 1.43 & 0.35 & 0.59 & 0.60 & 0.90 & 0.39 & 0.9 & 0.98 & 0.98 & 14 \\
\hline Thoothukudi & 1.22 & 0.94 & 0.48 & 0.01 & 0.27 & 0.28 & 0.29 & 0.27 & 0.4 & 0.49 & 0.47 & 7 \\
\hline The Nilgiris & - & - & - & 0.01 & 0.00 & 0.00 & 0.00 & 0.01 & 0.0 & 0.01 & 0.01 & 0.14 \\
\hline Kanyakumari & 0.01 & 0.04 & 0.02 & 0.02 & 0.01 & 0.02 & 0.04 & 0.03 & 0.0 & 0.02 & 0.02 & 0.33 \\
\hline District Average & 8.7 & 9.2 & 8.41 & 7.41 & 6.7 & 6.55 & 6.6 & 5.71 & 5.6 & 5.71 & 7.06 & \\
\hline
\end{tabular}


Table.4 Categorization of efficient zone

\begin{tabular}{|l|c|c|c|}
\hline Districts & RSI & RYI & Category \\
\hline Kancheepuram & $\mathrm{H}$ & $\mathrm{H}$ & MECZ \\
\hline Thiruvallur & $\mathrm{M}$ & $\mathrm{H}$ & MECZ \\
\hline Cuddalore & $\mathrm{L}$ & $\mathrm{H}$ & $\mathrm{ECZ}$ \\
\hline Vilupuram & $\mathrm{H}$ & $\mathrm{H}$ & $\mathrm{MECZ}$ \\
\hline Vellore & $\mathrm{H}$ & $\mathrm{M}$ & $\mathrm{ECZ}$ \\
\hline Thiruvannamalai & $\mathrm{H}$ & $\mathrm{M}$ & $\mathrm{ECZ}$ \\
\hline Salem & $\mathrm{M}$ & $\mathrm{M}$ & $\mathrm{ECZ}$ \\
\hline Namakkal & $\mathrm{H}$ & $\mathrm{M}$ & $\mathrm{ECZ}$ \\
\hline Dharmapuri & $\mathrm{M}$ & $\mathrm{H}$ & $\mathrm{MECZ}$ \\
\hline Krishnagiri & $\mathrm{M}$ & $\mathrm{M}$ & $\mathrm{ECZ}$ \\
\hline Coimbatore & $\mathrm{L}$ & $\mathrm{M}$ & $\mathrm{ECZ}$ \\
\hline Thiruppur & $\mathrm{L}$ & $\mathrm{M}$ & $\mathrm{ECZ}$ \\
\hline Erode & $\mathrm{H}$ & $\mathrm{L}$ & $\mathrm{NECZ}$ \\
\hline Thiruchirappalli & $\mathrm{M}$ & $\mathrm{M}$ & $\mathrm{ECZ}$ \\
\hline Karur & $\mathrm{M}$ & $\mathrm{H}$ & $\mathrm{MECZ}$ \\
\hline Perambalur & $\mathrm{L}$ & $\mathrm{M}$ & $\mathrm{ECZ}$ \\
\hline Ariyalur & $\mathrm{L}$ & $\mathrm{H}$ & $\mathrm{ECZ}$ \\
\hline Pudukkottai & $\mathrm{H}$ & $\mathrm{M}$ & $\mathrm{ECZ}$ \\
\hline Thanjavur & $\mathrm{L}$ & $\mathrm{H}$ & $\mathrm{ECZ}$ \\
\hline Thiruvarur & $\mathrm{L}$ & $\mathrm{H}$ & $\mathrm{ECZ}$ \\
\hline Nagapattinam & $\mathrm{L}$ & $\mathrm{H}$ & $\mathrm{ECZ}$ \\
\hline Madurai & $\mathrm{L}$ & $\mathrm{M}$ & $\mathrm{ECZ}$ \\
\hline Theni & $\mathrm{L}$ & $\mathrm{H}$ & $\mathrm{ECZ}$ \\
\hline Dindigul & $\mathrm{L}$ & $\mathrm{H}$ & $\mathrm{ECZ}$ \\
\hline Ramanathapuram & $\mathrm{L}$ & $\mathrm{L}$ & $\mathrm{HICZ}$ \\
\hline Virudhunagar & $\mathrm{L}$ & $\mathrm{L}$ & $\mathrm{HICZ}$ \\
\hline Sivagangai & $\mathrm{L}$ & $\mathrm{L}$ & $\mathrm{HICZ}$ \\
\hline Thirunelveli & $\mathrm{L}$ & $\mathrm{M}$ & $\mathrm{ECZ}$ \\
\hline Thoothukudi & $\mathrm{L}$ & $\mathrm{M}$ & $\mathrm{ECZ}$ \\
\hline The Nilgiris & $\mathrm{L}$ & $\mathrm{L}$ & $\mathrm{HICZ}$ \\
\hline Kanyakumari & $\mathrm{L}$ & $\mathrm{M}$ & $\mathrm{ECZ}$ \\
\hline
\end{tabular}

H-High; M-Medium; L-Low; MECZ- Most Efficient Cropping Zone; ECZ-Efficient Cropping Zone; NECZ-Not Efficient Cropping Zone; HICZ-Highly Inefficient Cropping Zone

Among other districts Vellore, Namakkal, Thiruvannamalai and Pudukkottai districts have high RSI and medium RYI values. Krishnagiri, Thiruchirappalli, Salem districts have medium RSI \& medium RYI. Cuddalore, Ariyalur, Thanjavur, Thiruvarur, Nagapattinam, Theni and Dindigul districts have low RSI \& high RYI. Coimbatore, Thiruppur, Perambalur, Madurai, Thirunelveli, Thoothukudi and Kanyakumari districts have low RSI \& medium RYI. So, these twenty one districts were categorized under Efficient Cropping Zone (ECZ). 
Erode district have high RSI and low RYI value, which was grouped under Not Efficient Cropping Zone (NECZ).

Rest of the four districts namely Ramanathapuram, Virudhunagar, Sivagangai and The Nilgiris have low RSI and low RYI values. These districts were identified as Highly Inefficient Cropping Zone (HICZ) for groundnut cultivation.

From this analysis, it is concluded that Kancheepuram district were found to be Highly Efficient Cropping Zone for groundnut cultivation. All soil, environmental and other factors in that area were highly suitable for groundnut cultivation. The technologies should be develop to further increase the productivity of groundnut. Crop loans and insurance for growing of groundnut will promote farmers to increase the area of cultivation and also minimize the risk.

\section{References}

Kanwar J. (1972). Cropping patterns, scope and concept, In. Proc. Symp, on Cropping Pattern in India, ICAR, New Delhi, 11-32.

Season and Crop Reports, Government of Tamil Nadu (2005 - 2016), Department of Economics and Statistics, Government of Tamil Nadu, Chennai.

\section{How to cite this article:}

Sanbagavalli, S., R. Tamilmozhi and Karthikeyan, R. 2020. Efficient Cropping Zone for Groundnut in Tamil Nadu. Int.J.Curr.Microbiol.App.Sci. 9(11): 2202-2207. doi: https://doi.org/10.20546/ijcmas.2020.911.263 